CELL ADHESION

\section{Destroying ties}

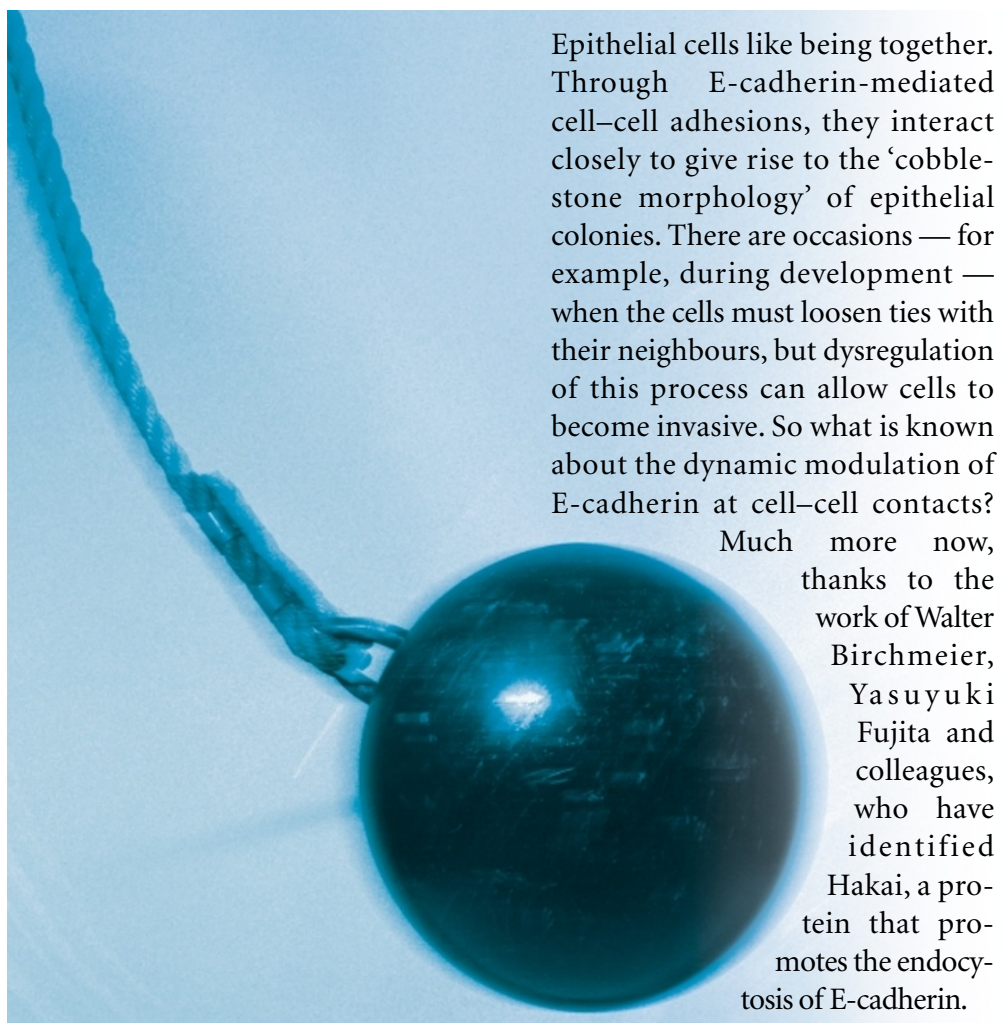

Activation of tyrosine kinases such as Src or Met is known to cause epithelial cells to develop a fibroblast-like morphology and scatter. E-cadherin is re-distributed into the intracellular compartment, indicating that it has been endocytosed. The authors first investigated whether E-cadherin was modified by these kinases, which it was. But not only was it phosphorylated (on tyrosines); it was also ubiquitylated.

Assuming that these modifications might alter the properties of $\mathrm{E}$ cadherin, Birchmeier's group carried out a two-hybrid screen, using tyrosine-phosphorylated cytoplasmic Ecadherin as bait, to look for potential binding partners. They subsequently cloned Hakai - Japanese for 'destruction' - which interacted specifically with E-cadherin in a phosphorylation-dependent manner through tyrosines 756 and 757.

In addition to its phosphotyrosine-binding domain, the predicted amino-acid sequence of Hakai contains a RING-finger at its amino terminus and a large percentage (35\%) of proline residues in its carboxyl terminus. In this respect, it resem- bles c-Cbl, an E3 ubiquitin ligase that recognizes tyrosine-phosphorylated receptor kinases. Modelling studies showed that a Src-homology-2 ( $\mathrm{SH} 2$ ) domain follows the combined RING and Zn-finger domain of Hakai. These occur in the reverse order in c-Cbl, but overall, the two domains seem to be arranged very similarly.

On the basis of this structural homology, the authors investigated Hakai's ability to mediate an increase in E-cadherin ubiquitylation to see if Hakai is also functionally related to c-Cbl. Using in vitro assays, they showed that, in the presence of Hakai, active Src and E2 ubiquitin-conjugating enzymes, Ecadherin was strongly ubiquitylated. They could also immunoprecipitate ubiquitylated E-cadherin from Hakai-transfected MDCK cells that were stimulated with scatter factor, and showed that the Ecadherin tyrosine residues that interacted with Hakai were crucial for ubiquitylation.

So what happens when E-cadherin is ubiquitylated? Cells transfected with Hakai were flatter and formed irregularly shaped colonies.

\section{CELL SIGNALLING}

\section{PYRIN fever}

The PYRIN domain is a recently discovered protein-protein interaction motif found at the amino termini of several proteins involved in apoptotic and inflammatory signalling pathways. Analysis of the human genome predicts a large family of PYRINcontaining proteins, some of which are thought to interact through their PYRIN domains. A report in the Journal of Biological Chemistry now describes a new member of the PYRIN-domain family, and identifies it as a novel activator of the NF- $\mathrm{KB}$ signalling pathway.

John Bertin and colleagues at Millennium Pharmaceuticals searched an expressedsequence-tag database to identify members of the nucleotide-binding site/leucine-rich repeat (NBS/LRR) family that have an amino-terminal PYRIN domain. They pulled out PYPAF1 ('PYRIN-containing Apaf1-like protein 1'), which also contains an NBS domain and a number of carboxy-terminal
LRRs. The authors then carried out a twohybrid screen and found that PYPAF1 interacts selectively with the PYRIN domain of a protein called ASC - an interaction that requires the PYRIN domain of PYPAF1.

Bertin's group next carried out cellular colocalization studies of the two proteins. Whereas PYPAF1 alone showed a broad cytoplasmic distribution, co-expression with ASC led to PYPAF1 being recruited to punctate structures in the cytoplasm. The PYRIN domain of PYPAF1 was needed for this recruitment, supporting the idea that this domain mediates the assembly of a PYPAF1/ASC complex.

But what does this complex do? Northern blotting analysis revealed that PYPAF1 is expressed mainly in peripheral blood leukocytes, suggesting a possible role in inflammatory signalling. Moreover, other NBS/LRR proteins are known to act as upstream regulators of NF- $\kappa \mathrm{B}$ signalling. So Bertin and colleagues studied the activation of NF- $\kappa B$ using a luciferase reporter plasmid.

Whereas PYPAF1 alone did not induce the activation of NF- $\mathrm{KB}$, expression of ASC alone could - but only at high concentrations.
However, co-expression of low levels of ASC (which did not activate NF- $\mathrm{KB}$ ) with PYPAF1 led to a 30-fold increase in NF- $\mathrm{KB}$ activity. Deletion of PYPAF1's PYRIN domain blocked this stimulation. Surprisingly, deletion of a carboxy-terminal region in PYPAF1 resulted in a twofold increase in the synergistic activation, and the authors suggest that the LRRs could act as a negative regulator of PYPAF1 activity. Finally, they showed that the NF- $\mathrm{KB}$ signalling occurs through the IKK complex, as dominantnegative versions of IKK- $\gamma$ and IKK-2 blocked the synergistic activation of NF- $\kappa \mathrm{B}$. The emerging story, then, is that PYRINcontaining proteins are important in innate immunity. Defects in the archetypal family member, pyrin, are associated with a rare inflammatory disease called familial Mediterranean fever. And as pyrin binds to ASC, the authors speculate that their findings point to a link between pyrin and the NF- $\mathrm{KB}$ signalling pathway.

(2) References and links Alison Mitchell ORIGINAL RESEARCH PAPER Manii, G. A. et al. PYPAF1: A PYRIN-containing Apaf1-like protein that assembles with ASC and regulates activation of NF-KB. J. Biol. Chem. 10 January 2002 (DOI 10.1074/jbc.M112208200) 